\title{
An insight in recurrent colon cancer: determining its risk factors and mechanisms to better assess and prevent this complication based on a case report
}

\author{
Mohamed Ali Mseddi ${ }^{1}$ \\ ${ }^{1}$ Habib Bourguiba Hospital
}

February 1, 2022

\begin{abstract}
The goal of the surgery is to eradicate the cancerous process without compromising patient's autonomy. Not leaving a residual tumour-spot that will offer a niche for local tumour recurrence is the surgeon's obsession. We highlight the mechanisms and risk factors to elaborate preventive and therapeutic means to combat this complication.
\end{abstract}

\section{Title}

An insight in recurrent colon cancer: determining its risk factors and mechanisms to better assess and prevent this complication based on a case report

\section{Corresponding author:}

Author: Mohamed Ali Mseddi

Affiliation: General surgery department, Habib Bourguiba hospital of Sfax, TUNISIA

E-mail: mseddibaka@gmail.com

\section{Key words}

Colon, cancer, recurrence, mechanism, prevention

\section{Introduction}

The surgical treatment of colon cancer is a therapeutic challenge. The goal of the resectional surgery is to eradicate the cancerous process without compromising the autonomy of the patient. The surgeon's obsession is not to leave a residual tumour spot that will offer a niche for local tumour recurrence. The diagnosis of tumour local recurrence is tricky. It must first be separated from metastatic regrowth or synchronous tumour development. This nosological separation is required in order to assess the prognosis and dictate the therapeutic course. Through this case report, we highlight the mechanisms and risk factors in order to elaborate preventive and therapeutic means to combat this complication.

\section{Case presentation:}

Patient H F, aged 81 year-old, whom father and brother passed away from colic cancer, was operated on 2017 for T3bN0M0 rectosigmoid junction cancer. He had a low segmental resection with a circular mechanical colorectal anastomosis protected by a right iliac ileostomy. The restoration of digestive continuity took place one year later. Three-years later, he consulted for the installation of weight loss, anorexia and tenacious constipation. The physical examination did not reveal any hepatomegaly or mass on palpation, nor ascites 
or adenomegaly. The rectal examination did not reveal any irregularity of the mucosa. He was charged by an abdominal scan, which demonstrated a lesion of $2 * 2 \mathrm{~cm}$ in sight of the surgical anastomosis (figure 1).

A colonoscopy reinforced this result by showing an irregular lesion of $2 \mathrm{~cm}$ occupying the anastomosis line (figure 2). It was confirmed as colon adenocarcinoma in pathology which is consistent with the histological type of first surgery. The patient underwent an anterior resection and protected mechanical colo-sus-anal anastomosis with no intraprocedural complications. Trimmed gross examination (figure 3) showed an irregular 2-centimeter-wide hard tumor developped into the suture line. The postoperative course was simple and he was discharged at fifth-day postoperatively.

\section{Discussion:}

The diagnosis of tumour local recurrence (LR) is tricky. It must first be separated from metastatic regrowth or synchronous tumour development. This nosological separation is required in order to assess the prognosis and dictate the therapeutic course.

Local recurrence is defined as tumor regrowth in and around the tumor bed. There are two types depending on the location in relation to the operative area: extramural recurrence if it is in the perianastomotic tissues outside the bowel wall including peri-colic fat, adjoining mesentery and lymph nodes or intramu$\mathrm{ral} /$ anastomotic recurrence if it in the suture or staple line of the anastomosis[1,2]. This dichotomy is irrelevant as the preponderance of anastomotic recurrences originate from perianastomotic soft tissue with subsequent growth in the staple line[1].

Most of the local recurrences that occur after 2-year lag period from previous surgery should be considered as metastatic recurrences or as second primary lesion[3].

However, synchronous cancer diagnosis can be made when it occurs simultaneously or within 6 months from the initial diagnosis and the histopathological criteria of Warren and Gates are respected: Each tumor has to show a clear out line of malignancy, has to be clearly distinct from the other, and must not be the metastatic expression of the other.

Most postoperative recurrences in patients with resected colon cancer occur during the first five years[4]. Recurrences are mostly metastatic ( $80 \%$ of cases), and occur in nearly $80 \%$ of cases in the first three years after surgery with a peak between 6-12 months. The more advanced the initial stage, the earlier they occur [5,6]. Although, LR is infrequent, it occupies the third rank of all recurrences after liver metastasis and lung metastasis[7]. Isolated anastomotic recurrence is uncommon[8]. In almost $50 \%$ of cases, these local recurrences are associated with metastatic spread[5].

Colonoscopy seems to be the means of screening for LR of choice biology is not relevant in this application. The utility of tumour markers appears to be low in the screening of LR. In fact, carcinoembryonic antigen (CEA) testing is most sensitive for hepatic or retroperitoneal metastasis and relatively insensitive for local, pulmonary, or peritoneal involvement[9]. CEA monitoring in which the primary tumor produced no elevation in the serum CEA level is not useful for the early detection of recurrent disease[10].

However, imaging allows a histological test to be omitted. When a tumour-like image occupying the operative scar is encountered in the course of morphological investigations, imaging can be used to postpone histological evidence with high reliability if it meets the following criteria: Perianastomotic or eccentric masses on computed tomography (CT) with corresponding perianastomotic or eccentric fluorodeoxyglucose (FDG) uptake on positron emission tomography (PET) are highly predictive of staple line recurrence. However, Back ground, diffuse, curvilinear, and focal patterns of FDG uptake, regardless of intensity of FDG uptake, do not correlate with recurrence in the absence of a mass on CT[2]. the results of a recent retrospective study defining these radiological diagnostic criteria of anastomotic recurrences are confirmed by histological study of a surgical resection specimen or on per-colonoscopic biopsy support this approach to management. FDG PET/CT interpretation yielded sensitivity, specificity, positive predictive value, negative predictive value, and accuracy results of $100 \%, 97.1 \%, 81.8 \%, 100 \%$, and $97.5 \%$ respectively. 
Factors that have been associated with an increased risk of developing LR in patients with colon cancer are: more advanced disease stage or tumour grade, lymphovascular invasion, involved resection margins, left sided tumours and where the tumour causes obstruction, perforation, invasion of adjacent structures[5].This can could be explained by residual tumoral cells during incomplete surgery not following carcinological constraints or due to extension spillage of tumoral juice into the peritoneal cavity if a perforation occurred spontaneously or when manipulating the neoplasm. Double stapling technique has a higher risk of anastomotic recurrence than hand-sewn anastomosis[11]. Postoperative intra-abdominal infection increases angiogenesis and tumor recurrence after surgical excision of tumor anastomotic break down in the postoperative period either clinically or radiologically[12], but a recent anastomotic dehiscence only favours rectal cancers as was concluded in a retrospective study where such post-operative complication double LR risk[13]. LR occurs more in distally located tumors: LR affects $12 \%$ of colonic cancers, whereas it affects $25-35 \%$ of rectal cancers[5]. Certain histological tumour characteristics are prone to recurrence such as as: moderately or poorly differentiated tumoral cells, mucinous type[6]. Still, a transplant from a distant metastasis should be considered, especially since systemic metastasis is more frequent.

A review of the literature identified the following mechanisms:

- free intraluminal cancer cells of colonic origin penetrate through watertight anastomoses, implant on the anastomotic surface, and initiate tumor regrowth[14]. In a retrospective clinical study focusing on whether carcinoma cells were present inside the distal ring of clamped colon conducted that cancer cells were retrieved in $70 \%$ from the irrigation solution applied on the ring of colon tissue where the distal clamp was applied[15]. Even if exfoliated cells could be found in case of a large tumor more easily, no association was found between the presence of exfoliated cells and neither the type of tumor nor the depth of intestinal wall infiltration[16]. In addition, number of viable tumor cells did not correlate with the stage, differentiation, diameter or fixity of the tumor[15].

- presence of normal appearing colonocytes within proximity of the primary bowel but with malignant potential as was confirmed by a recent study: margins adjacent to the resected primary tumor had been initially examined and found free of dysplastic or atypical cells histologically, were re-examined after the patient presented with recurrent tumor. In these patients the mucosal cells adjacent to the site of the primary lesion were checked for the expression of tumor antigen. By using these antibodies in examining post-operative colon cancer specimens, this antigen expression was also present in phenotypically normal appearing colonocytes adjacent to the primary malignancy. The antigen in the anastomotic recurrence was then compared to that in the primary cancer as well as the altered colonocytes and found to be identical[17]. The initial groups of cells that complete the transformation to a fully malignant phenotype probably suppress surrounding premalignant cells from further transformation on a local immunogenic basis. Removal of the primary lesion may eliminate this suppressive effect and allow premalignant colonocytes expressing antigen, to progress to the fully malignant phenotype[18]. If these potentially malignant cells are incorporated inadvertently into suture points used to establish digestive continuity, they will form a niche for carcinogensis.

- presence of a small synchronous neoplasia, unknown at the moment of resection but close to the anastomosis, especially a small, flat lesion[3].

- the continuous proliferative stimulus due to chronic inflammation could favor neoplastic growth by healing impairment and inflicting alteration of mucosal biological properties[3]. In particular in right hemicolectomies, the absence of the ileocolic valve can influence the biology of the anastomotic mucosa, by diversion colitis[3].

We therefore determine the following preventive means: intraluminal irrigation with the cancericidal agent such $5 \%$ povidone-iodine solution of the colon is practiced with reference to the latest recommendations on the quality criteria for the surgical removal of rectal cancers[19]. This procedure decreased suture line recurrence rates from 10\%-16\% to 2\% -3\%[8]. Such prophylactic procedure is commonly used during anterior resection for rectal cancer where rectal washout reduces by half the risk of local recurrence as stated by a recent metanalysis[20]. Intestinal irrigation should be carried for preferrably 5 minutes, although longer periods (15 minutes) seem to give better results; but periods longer than 30 minutes damage the cells of 
the normal colic mucosa irreversibly[3]. Some authors suggest using low-osmolarity fluid (water) instead of normal saline in order to lyse cancer cells during surgery[1]. But the effectiveness of such a manoeuver is still debated. Recent literature review focusing on repeated anastomotic recurrences found that tumoral anastomotic relapse occurred despite colorectal irrigation in half of patients[8]. Also, intestinal preparation with PEG solution seems to reduce the risk of the implantation of exfoliated cells present in the colonic lumen[2]. Removal of the cancer should be carried with a minimum $5 \mathrm{~cm}$ distal and proximal margin, assorted with en bloc removal of the attached mesocolon. No touch technique and primary vessel ligation should be emphazied.

As opposed to synchronous tumours which demand extensive salvage surgery, patients with isolated perianastomotic or limited nodal recurrence in the mesentery may be potentially curative by radical resection in addition to adjuvant chemotherapy. The procedure of choice is segmental colectomy complying habitual carcinological exigencies, as stated above.

\section{Conclusions:}

Local recurrences of colon cancers is a concerning complication of most surgeons. A more insight of its mechanism allows undertaking effective preventive procedures to reduce its occurance. Colonoscopy or, if inocclusive exploration, conjunction of PET/CT should rule out tumoral relapse at a more indolent stage, thus allowing a curative treatment. Both exfoliated tumoral cells, premalignant normal looking colonocytes and incomplete surgery represent the major mechanism of tumoral relapse. Factors associated with an increased risk of developing local recurrences must be recognized by surgeons to better follow up at-risk patients. Intraluminal irrigation with the cancericidal agent and pre-operative intestinal cleansing decrease intramural recurrence. While following carcinological constraints should decrease tumoral recurrence. Salvage surgery remains the mainstream treatment for recurrent colon cancer.

\section{Abbreviations:}

LR: local recurrence

CEA: carcinoembryonic antigen

CT: computed tomography

FDG: fluorodeoxyglucose

PET: positron emission tomography

\section{Declaration of Competing Interest}

The author certifies that there is no conflict of interest regarding the material discussed in the manuscript.

\section{Funding sources}

The author declares that this research didn't receive any specific grant from funding agencies in the public, commercial, or not-for-profit sectors.

\section{Ethical approval}

Ethical approval has been exempted by our institution because this is a case report and no new studies or new techniques were carried out.

The author is accountable for all aspects of the work in ensuring that questions related to the accuracy or integrity of any part of the work are appropriately investigated and resolved.

\section{Consent}

Written informed consent was obtained from the patient for publication of this case report and accompanying images. A copy of the written consent is available for the Editor-in-Chief of this journal on request.

\section{Author contribution}


Mohamed Ali MSEDDI: drafting and revising the manuscript, literature research.

\section{Registration of research studies}

Not applicable.

\section{Guarantor}

The guarantor for this case report is Atef MEJRI.

\section{Provenance and peer review}

Not commissioned, externally peer-reviewed.

\section{Acknowledgements}

We would like to express our deep gratitude to all contributors for the suggestion and correction in making this article.

\section{References:}

[1] A.M. Abulafi, N.S. Williams, Local recurrence of colorectal cancer: the problem, mechanisms, management and adjuvant therapy, Br J Surg. 81 (1994) 7-19. https://doi.org/10.1002/bjs.1800810106.

[2] P.B. Shyn, R. Madan, C. Wu, Ş.M. Erturk, S.G. Silverman, PET/CT Pattern Analysis for Surgical Staple Line Recurrence in Patients With Colorectal Cancer, American Journal of Roentgenology. 194 (2010) 414-421. https://doi.org/10.2214/AJR.09.2892.

[3] P. Genova, V.D. Palumbo, A.I. Lo Monte, C. Cipolla, G. Genova, Unexplained neoplastic anastomotic recurrence after right hemicolectomy: a case report, J Med Case Reports. 14 (2020) 196. https://doi.org/10.1186/s13256-020-02529-z.

[4] T. Akiyoshi, Y. Fujimoto, T. Konishi, H. Kuroyanagi, M. Ueno, M. Oya, S. Miyata, T. Yamaguchi, Prognostic factors for survival after salvage surgery for locoregional recurrence of colon cancer, Am J Surg. 201 (2011) 726-733. https://doi.org/10.1016/j.amjsurg.2010.03.003.

[5] Incidence and patterns of recurrence after resection for cure of colonic cancer in a well defined population - PubMed, (n.d.). https://pubmed.ncbi.nlm.nih.gov/16804870/ (accessed December 19, 2021).

[6] E. Vakiani, R.H. Shah, M.F. Berger, A.P. Makohon-Moore, J.G. Reiter, I. Ostrovnaya, M.A. Attiyeh, A. Cercek, J. Shia, C.A. Iacobuzio-Donahue, D.B. Solit, M.R. Weiser, Local recurrences at the anastomotic area are clonally related to the primary tumor in sporadic colorectal carcinoma, Oncotarget. 8 (2017) 42487-42494. https://doi.org/10.18632/oncotarget.17200.

[7] Patterns of recurrence after curative resection of carcinoma of the colon and rectum - PubMed, (n.d.). https://pubmed.ncbi.nlm.nih.gov/1729745/ (accessed December 19, 2021).

[8] G. Conzo, C. Mauriello, C. Gambardella, F. Cavallo, E. Tartaglia, S. Napolitano, L. Santini, Isolated repeated anastomotic recurrence after sigmoidectomy, WJG. 20 (2014) 16343. https://doi.org/10.3748/wjg.v20.i43.16343.

[9] An evaluation of the carcinoembryonic antigen (CEA) test for monitoring patients with resected colon cancer - PubMed, (n.d.). https://pubmed.ncbi.nlm.nih.gov/8141873/ (accessed December 19, 2021).

[10] M.W. Wichmann, U. Lau-Werner, C. Müller, H.M. Hornung, P. Stieber, F.W. Schildberg, Colorectal Cancer Study Group, Carcinoembryonic antigen for the detection of recurrent disease following curative resection of colorectal cancer, Anticancer Res. 20 (2000) 4953-4955.

[11] B. Anderberg, P. Enblad, R. Sjödahl, J. Wetterfors, Recurrent rectal carcinoma after anterior resection and rectal stapling, British Journal of Surgery. 71 (1984) 98-100. https://doi.org/10.1002/bjs.1800710206. 
[12] Postoperative intra-abdominal infection increases angiogenesis and tumor recurrence after surgical excision of colon cancer in mice - PubMed, (n.d.). https://pubmed.ncbi.nlm.nih.gov/19767043/ (accessed December 19, 2021).

[13] J.M. Eberhardt, R.P. Kiran, I.C. Lavery, The impact of anastomotic leak and intra-abdominal abscess on cancer-related outcomes after resection for colorectal cancer: a case control study, Dis Colon Rectum. 52 (2009) 380-386. https://doi.org/10.1007/DCR.0b013e31819ad488.

[14] Y. Kluger, Y. Galili, J. Yossiphov, A. Shnaper, G. Goldman, M. Rabau, Model of implantation of tumor cells simulating recurrence in colonic anastomosis in mice, Dis Colon Rectum. 41 (1998) 1506-1510. https://doi.org/10.1007/BF02237297.

[15] T.-C. Hsu, M.-J. Chen, Presence of colon carcinoma cells at the resection line may cause recurrence following stapling anastomosis, Asian Journal of Surgery. 41 (2018) 569-572. https://doi.org/10.1016/j.asjsur.2018.01.006.

[16] P. Genova, C. Cipolla, G. Genova, G. Graceffa, S. Vieni, What is the Meaning of an Early Anastomotic Recurrence after Curative Right Hemicolectomy? A Synchronous, Metachronous, or What Else?, The American Surgeon. 85 (2019) 300-302. https://doi.org/10.1177/000313481908500611.

[17] A. M., C. J., C. G., S. O., B. J., D. A., S. J., C. C., K. A., P. J., A. P., W. X., M. E., The Cause and Prevention of Anastomotic Recurrence following Colectomy: An Immunohistochemical Approach for Detecting Transforming Colonocytes, J. Cancer. 5 (2014) 784-789. https://doi.org/10.7150/jca.9485.

[18] G.T. Motz, G. Coukos, Deciphering and reversing tumor immune suppression, Immunity. 39 (2013) 61-73. https://doi.org/10.1016/j.immuni.2013.07.005.

[19] V. Bridoux, C. de Chaisemartin, L. Beyer, N. Goasguen, C. Sabbagh, N. Guedj, P. Dartigues, A. Bardier, Recommandations pour la pratique clinique Cancer du rectum: Question 2 Quels sont les critères de qualité de l'exérèse chirurgicale ?, Colon Rectum. 10 (2016) 12-27. https://doi.org/10.1007/s11725-015-0617-z.

[20] C. Zhou, Y. Ren, J. Li, X. Li, J. He, P. Liu, Systematic review and meta-analysis of rectal washout on risk of local recurrence for cancer, J Surg Res. 189 (2014) 7-16. https://doi.org/10.1016/j.jss.2014.01.030.

\section{FIGURES:}

Figure 1: Representive abdominal CT slice associated with this case

Figure 2: The corresponding colonoscopic highlighting the tumor developed into the digestive anastomosis

Figure 3 : Trimmed gross specimen showing the tumor (red arrow head) developped into the suture line (yellow arrow head) 

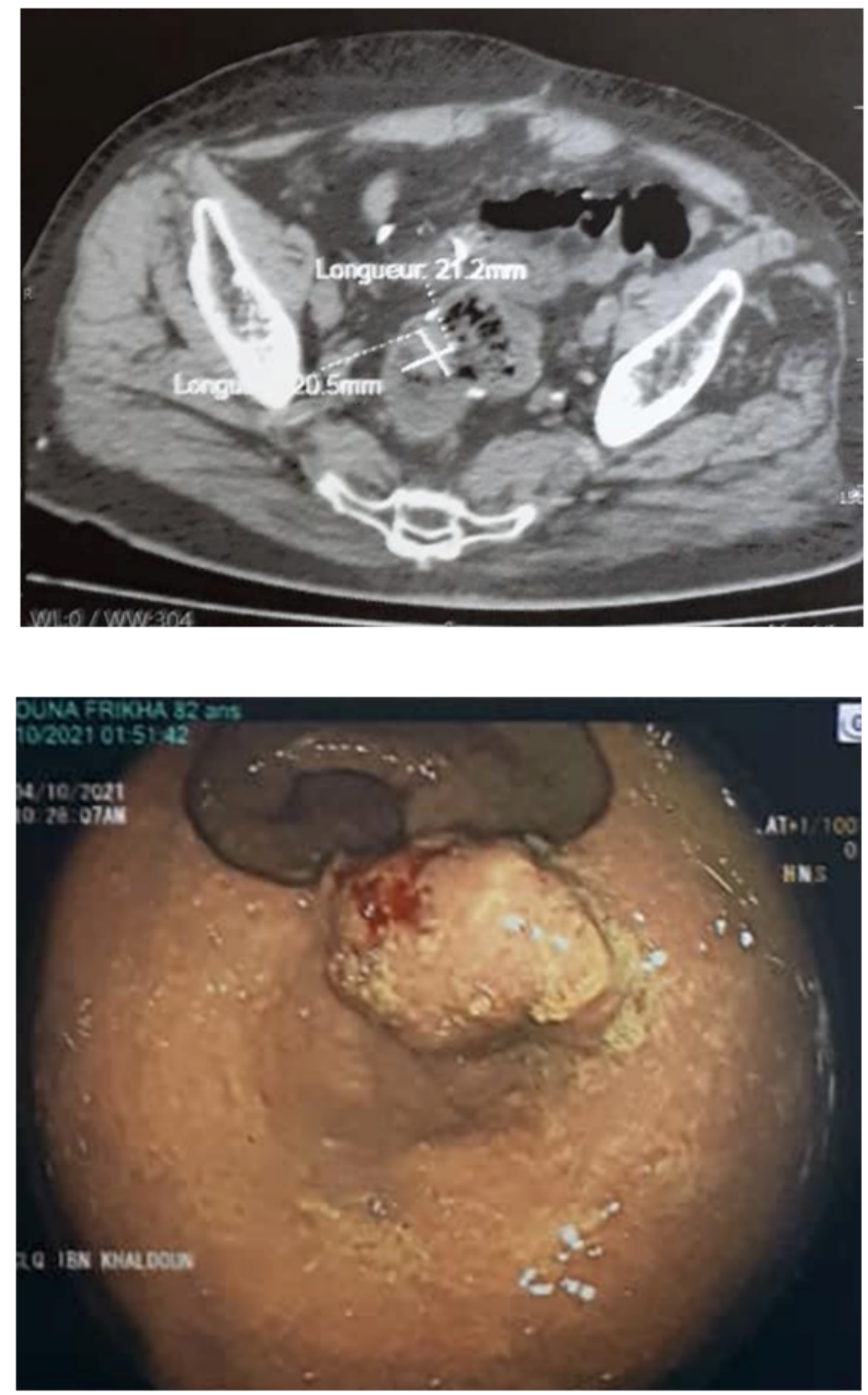


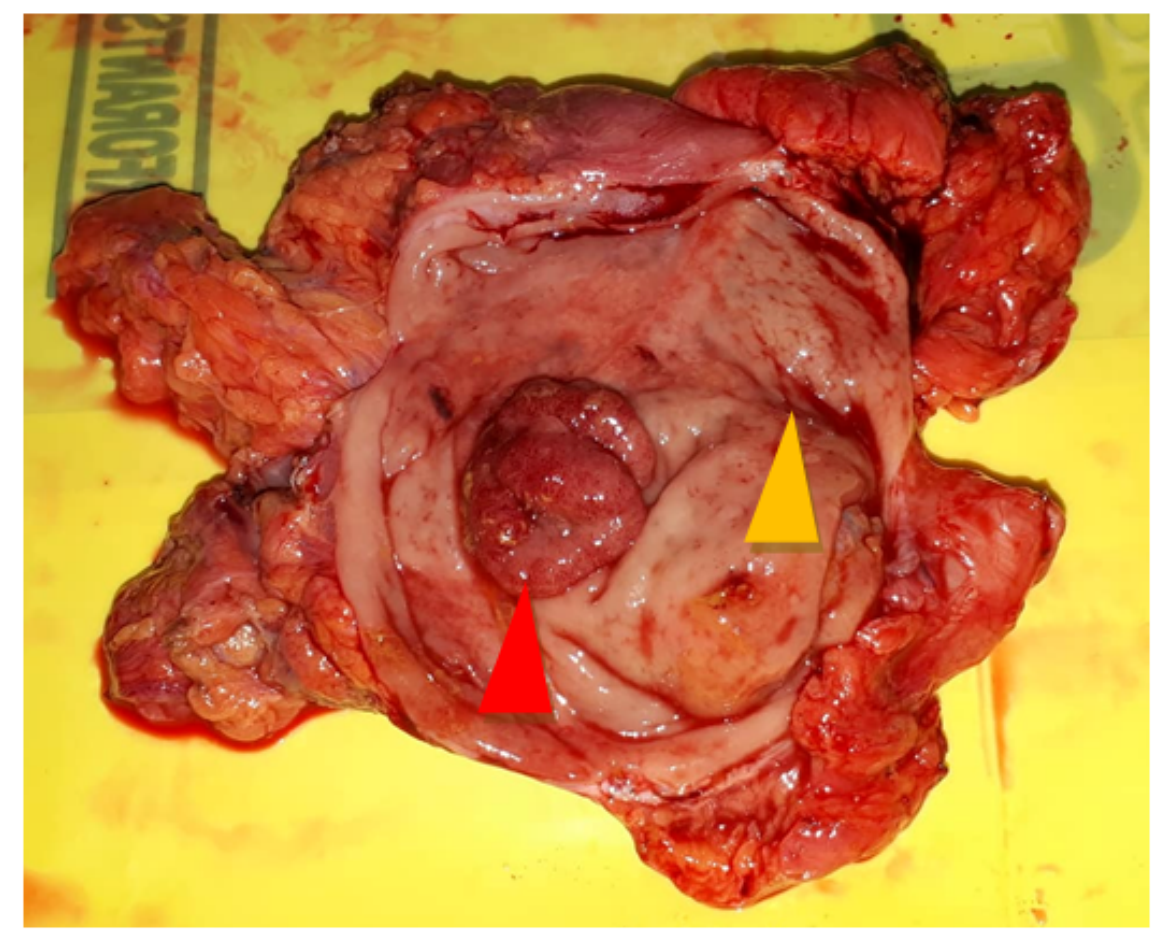

